\title{
Robust QFT Controller Design for Positioning a Permanent Magnet Stepper Motors
}

\author{
Hazem I.Ali
}

\begin{abstract}
In this paper, an application of quantitative feedback theory QFT for permanent magnet stepping motor is presented. This approach has been considered in order to assure high control performance of the system. The QFT controller is used to design a simple, low order, and robust position control for a permanent magnet stepping motor. A two-phase motor is considered in this paper. Also the QFT controller is designed to give a robust stability and performance (disturbance rejection, reference tracking) with the presence of system parameters uncertainty. The objectives of the controller are verified in simulation.
\end{abstract}

Index Terms-Robust control, QFT, Nonlinear system, Stepping motor

\section{INTRODUCTION}

It is well known that the stepper motor is an electromagnetic actuator that converts digital pulse inputs to analog shaft motion outputs. It rotates by a specific number of degrees in response to an electrical pulse input; therefore the stepper motor is used in digital control systems. Stepper motors are widely used in our daily life. They are used in practical applications that require incremental motion such as printers, tape drives, hard drives in PC's, machine tools, process control systems, X-Y records, and robotics [1]. Also the stepper motors are nonlinear and uncertain electromechanical incremental actuators. Their ability to provide accurate control over speed and position combined with their small size and relatively low cost make stepper motors a popular choice in range of applications. In particular, permanent magnet stepper motors deliver higher peak torque per unit weight and have a higher torque to inertia than DC motors. Furthermore, they are more reliable and, being brushless machines, require less maintenance [2].

The control system is one of the most important elements in stepping motor applications. The control systems of stepping motors are classified into open loop and closed loop schemes. In the open loop control scheme there is no feedback information of position to the controller and therefore it is imperative that the motor must respond correctly to each excitation change. If the excitation changes are made too quickly, the motor is unable to move to the new demanded position and consequently there is a permanent error in the actual position compared to the position expected by the controller. The timing of phase control signals for

Control and systems Engineering Department, University of Technology, Iraq-Baghdad

Hazemcontrol2001@yahoo.com optimum open-loop performance is reasonably straightforward, if the load parameters remain constant. However, in the applications where the load varies significantly, the timing must be set for the worst conditions (largest load) and the control scheme is then non optimal for all other loads [3, 4].

In more sophisticated methods of the open loop control, the variations of load and friction torques with speed can also be taken into account. Moreover, if higher resolution is needed the microstepping drive method can be used, but then the inaccuracy of the system increases due to the nonlinearity of microstepping. If high accuracy is needed, the closed loop control scheme is recommended. In closed loop stepping motor systems the instantaneous rotor position is detected via a feedback sensor and sent to the control unit. The general block diagram of the closed loop scheme is presented in figure (1).

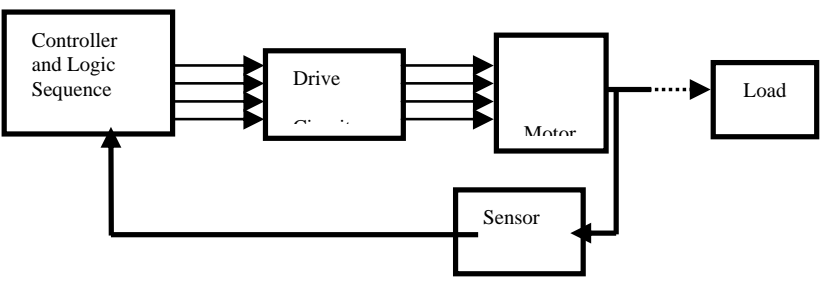

Fig. 1 Block diagram of closed loop stepping motor

Essentially, there are several approaches how to design the closed loop control system. Most used method is based on a switching angle (lead angle) and it is used in applications with rotary stepping motors coupled with rotary encoders, where the information about position of the rotor against the stator is measured. However, the linear motor drive uses the rotary stepping motor with a lead screw.

In this application, it is impossible to measure the displacement of the rotor due to the motor structure. The linear optical encoder is used to measure the linear displacement. This gives a better precision of the linear drive, because a sensor directly measures the control variable. It is essentially true that for many applications the open loop control is entirely adequate and choosing a closed loop system would be an expensive luxury. Nowadays, the use of microprocessors can decrease the price and if the high accuracy and high reliability of the positioning system is required, the closed loop control looks very attractive [3]

Two types of stepper motors are widely used, the variable reluctance type and the permanent magnet type. In this paper, the permanent magnet type is used. These types have higher inertia and therefore slower acceleration than variable reluctance types. They also produce more torque per ampere stator current than the variable reluctance [1]. 
In this paper the quantitative feedback theory (QFT) is applied to obtain a simple, low order and robust position controller for a permanent magnet stepping motor with the presence of system parameters uncertainty.

\section{SYSTEM MODELING}

This section will deal with building a mathematical model of the linear motor drive. Physical modeling approach is used for constructing the system model. The principle of physical modeling is to divide the properties of the system to subsystems whose behaviors are known. For a technical system, this means that the laws of nature describing the subsystems are used in general [5].

Basically, the model of the permanent magnet stepping motor consists of two parts, an electrical and a mechanical part [6]. The permanent magnet stepper motor dynamical model includes nonlinearities and contains some physical parameters. The values of physical parameters are not exactly known and can be subjected to some variations, so the model is not very easy to handle for control synthesis. Figure (2) shows the model for a permanent magnet stepping motor. The model has two phases denoted by $A$ and $B$. The rotor has $(2 \mathrm{Nr})$ magnetic poles, while the stator has a set of identical poles and windings equally arranged at intervals of $(\lambda)$ [4].

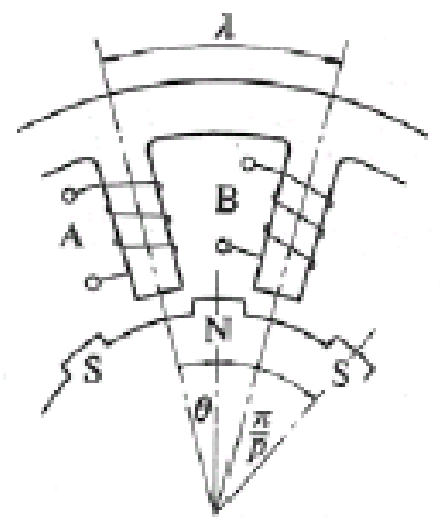

Fig. 2 Permanent magnet stepper motor

The dynamic equations for the motion of the rotor are developed. Let $\mathrm{T}$ be the developed torque by motor windings, $\mathrm{J}$ the inertia of the rotor, D the coefficient of viscous friction, $T_{F}$ represents the detent and the coulomb frictional torque, and $\theta$ is the rotor angular position. Then, the dynamics of the rotor can be expressed by the following equation:

$$
T=J \frac{d^{2} \theta}{d t^{2}}+D \frac{d \theta}{d t}+T_{F}
$$

The torques produced by windings $\mathrm{A}$ and $\mathrm{B}$ are given by:

$$
\begin{gathered}
T_{A}=-N_{r} n \Phi_{M} i_{A} \sin \left(N_{r} . \theta\right) \\
T_{B}=-N_{r} n \Phi_{M} i_{B} \sin \left(N_{r} .(\theta-\lambda)\right)
\end{gathered}
$$

Where $i_{A}, i_{B}$ are the currents in windings $\mathrm{A}$ and $\mathrm{B}, N_{r}$ is the number of the rotor teeth, $n \Phi_{M}$ is the flux linkage, $\theta$ is the rotational angle of the rotor and $\lambda$ is the tooth pitch in radians.

The mechanical part of the permanent magnet stepper motor model can be expressed by an equation derived from equations (1), (2) and (3):

$$
\begin{aligned}
& J \frac{d^{2} \theta}{d t^{2}}+D \frac{d \theta}{d t}+N_{r} n \Phi_{M} i_{A} \sin \left(N_{r} \theta\right)+ \\
& N_{r} n \Phi_{M} i_{B} \sin \left(N_{r}(\theta-\lambda)\right)+C \operatorname{sign}\left(\frac{d \theta}{d t}\right)+T_{L}=0
\end{aligned}
$$

Where $J$ denotes the moment of rotor inertia $\left(K g . m^{2}\right), D$ denotes the viscous damping coefficient (N.m.S.rad ${ }^{-1}$ ), C represents the coulomb friction coefficient, and $T L$ is the load torque $[4,6]$.

The electrical part of a permanent magnet stepper motor model is described by voltage equations for the stator windings.

$$
\begin{gathered}
V-r i_{A}-L \frac{d i_{A}}{d t}-M \frac{d i_{B}}{d t}+\frac{d}{d t}\left(n \Phi_{M} \cos \left(N_{r} \theta\right)\right)=0 \\
V-r i_{B}-L \frac{d i_{B}}{d t}-M \frac{d i_{A}}{d t}+\frac{d}{d t}\left(n \Phi_{M} \cos \left(N_{r}(\theta-\lambda)\right)\right)=0
\end{gathered}
$$

Where $V$ is the DC terminal voltage supplied to the stator windings (volt), L denotes the self-inductance of each stator phase $(\mathrm{mH}), M$ represents the mutual inductance between phases $(\mathrm{mH})$ and $r$ is stator circuit resistance (ohm). Thus, the complete model of the permanent magnet stepping motor consists of the rotor dynamic equation (4) and differential equations for current equation (5) and (6). Those equations are nonlinear differential equations. Since it is very difficult to deal with nonlinear differential equations analytically, linearization is needed. Linearization is made with aid of a new variable $\delta \theta$, which represents the deviation of the angle from the equilibrium position. The deviation is a function of time $t$ and very small in magnitude. Figure (3) shows two stator phases, which carry the stationary current $I_{o}$ in a direction to create south pole. The equilibrium position of the stator is then at $\theta=\frac{\lambda}{2}$ [4].

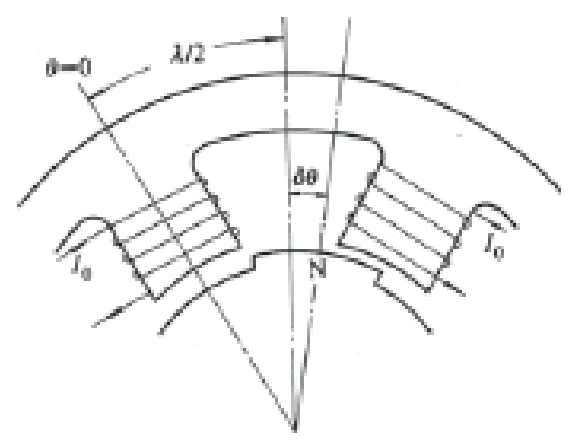

Fig. 3 Two stator phases of motor

When the rotor oscillates about its equilibrium position, the currents in both motor windings will deviate from the stationary value $I_{o}$ by $\delta i_{A} a n d \delta i_{B}$ and the angular rotor position can be expressed by:

$$
\theta=\frac{\lambda}{2}+\delta \theta
$$

The current in both windings are expressed as follows:

$$
i_{A}=I_{o}+\delta i_{A}
$$




$$
i_{B}=I_{o}+\delta i_{B}
$$

Then the nonlinearities expressed by sin and cosine functions in equations (4), (5) and (6) will be approximated with knowledge of trigonometric identities and when $N_{r} \delta \theta \quad$ is small angle: $\cos \left(N_{r} \delta \theta\right) \cong 1$ and $\sin \left(N_{r} \delta \theta\right)=N_{r} \delta \theta$.

After substituting those approximations into the motor equations, the linearized model is obtained in the following form (more detailed description is given in [4]):

$$
\begin{gathered}
J \frac{d^{2}(\delta \theta)}{d t^{2}}+D \frac{d(\delta \theta)}{d t}+2 N^{2}{ }_{r} n \Phi_{M} I_{o} \cos \left(\frac{N_{r} \lambda}{2}\right) \delta \theta+ \\
N_{r} n \Phi_{M} \sin \left(\frac{N_{r} \lambda}{2}\right)\left(\delta i_{A}-\delta i_{B}\right)+C \operatorname{sig} n\left(\frac{d \theta}{d t}\right)=0 \\
r \delta i_{A}+L \frac{d\left(\delta i_{A}\right)}{d t}+M \frac{d\left(\delta i_{B}\right)}{d t}-N_{r} n \Phi_{M} \sin \left(\frac{N_{r} \lambda}{2}\right) \frac{d(\delta \theta)}{d t}=0 \\
r \delta i_{B}+L \frac{d\left(\delta i_{B}\right)}{d t}+M \frac{d\left(\delta i_{A}\right)}{d t}+N_{r} n \Phi_{M} \sin \left(\frac{N_{r} \lambda}{2}\right) \frac{d(\delta \theta)}{d t}=0
\end{gathered}
$$

Where: $\sin \left(\frac{N_{r} \lambda}{2}\right)$, and $\cos \left(\frac{N_{r} \lambda}{2}\right)$ are constants.

The permanent magnet stepping motor transfer function is derived from equations (10), (11) and (12) with the aid of Laplace transform. The coulomb friction coefficient $C$ is considered to be zero. The resulting form of the transfer function in two-phase excitation in the voltage source drive is:

$$
\frac{\Theta_{o}}{\Theta_{i}}=\frac{\frac{r}{L} w^{2}{ }_{n p}}{s^{3}+\left(\frac{r}{L_{p}}+\frac{D}{J}\right) s^{2}+\left(\frac{r D}{L_{p} J}+w^{2}{ }_{n p}\left(1+k_{p}\right)\right) s+\left(\frac{r}{L_{p}}\right) w^{2}}
$$

Where

$$
L_{p}=L-M, w^{2}{ }_{n p}=\frac{2 N^{2}{ }_{r} n \Phi_{M} I_{o} \cos \left(\frac{N_{r} \lambda}{2}\right)}{J}, k_{p}=\frac{n \Phi_{M} \sin ^{2}\left(\frac{N_{r} \lambda}{2}\right)}{L_{p} I_{o} \cos \left(\frac{N_{r} \lambda}{2}\right)}
$$

, $\Theta_{o}$ is the Laplace transform of the actual rotor position,

$\Theta_{i}$ represents the Laplace transform of the demanded position and $s$ is the Laplace operator. Table (1) defines the model parameters and summarizes the nominal values of all parameters and their variations used in the controller design [7]. Figure (4) shows the frequency characteristics of the system with all parameters uncertainty.

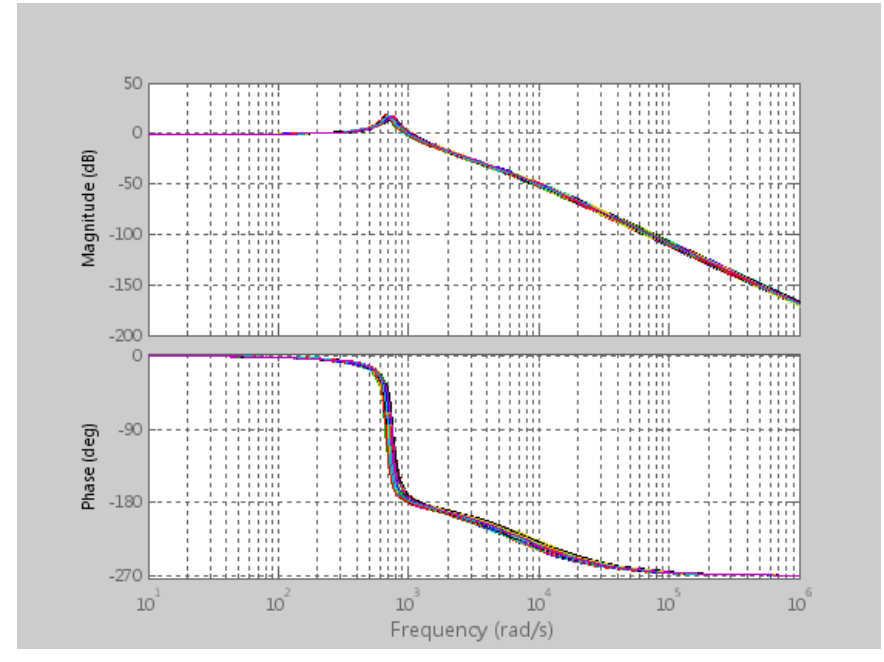

Fig. 4 Frequency characteristics of the system with all parameters uncertainty

It is clear the poor stability and performance of the system. Figure (5) shows the time response characteristics of the system with all parameters uncertainty.

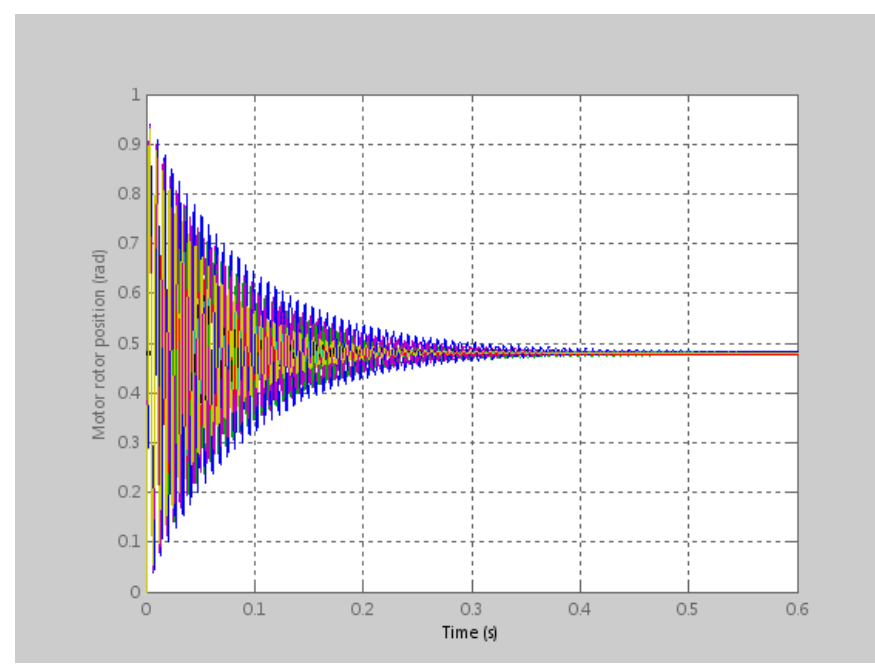

Fig. 5 Closed loop time response characteristics of the system with all parameters uncertainty

TABLE I: THE NOMINAL MODEL PARAMETERS AND THEIR RANGE

\begin{tabular}{|l|c|c|c|}
\hline \multicolumn{1}{|c|}{ PARAMETER } & $\begin{array}{c}\text { MINIMUM } \\
\text { VALUE }\end{array}$ & $\begin{array}{c}\text { NOMINAL } \\
\text { VALUE }\end{array}$ & $\begin{array}{c}\text { MAXIMUM } \\
\text { VALUE }\end{array}$ \\
\hline Stator resistance (r) ohm & 29.7 & 33 & 36.3 \\
\hline Self inductance (L) $\mathrm{mH}$ & 4.86 & 5.4 & 5.94 \\
\hline Matual inductance (M) mH & 0.36 & 0.4 & 0.44 \\
\hline $\begin{array}{l}\text { Rotor inertia (J) } \\
g . \mathrm{cm}^{2}\end{array}$ & & 0.16 & \\
\hline Number of rotor teeth $\left(N_{r}\right)$ & & 6 & \\
\hline $\begin{array}{l}\text { Viscous friction (D) (N.m.s/rad)* } \\
10^{\wedge}-5\end{array}$ & 1.215 & 1.35 & 1.485 \\
\hline $\begin{array}{l}\text { Tooth pitch }(\lambda) \\
\text { rad }\end{array}$ & & $\pi / 12$ & \\
\hline Stationary current $\left(I_{o}\right)$ Amper & & 0.15 & \\
\hline $\begin{array}{l}\text { Flux linkage } \\
\left(n \Phi_{M}\right) T . m^{2}{ }^{*} 10^{\wedge}-3\end{array}$ & 1.08 & 1.2 & 1.32 \\
\hline
\end{tabular}




\section{QFT CONTROL METHODOLOGY}

The QFT design methodology is quite transparent, allowing the designer to see the necessary trade- offs to achieve the closed loop system specifications. The basic steps of the procedure are:

i) Plant model (with uncertainty), templates generation and nominal plant selection.

ii) Performance specifications.

iii) QFT bounds.

iv) Loop shaping the controller.

v) Pre-filter synthesis.

vi) Simulation and design validation.

The plant dynamics to be controlled is described by a linearized model, which represents the nominal model of the system. The parameter uncertainty of the system is taken at every frequency of interest $\left(w_{i}\right)$, that is to say the plant uncertainty templates, which are set of complex numbers representing the frequency response of the family of uncertain plants at a fixed frequency. Figure (6) shows the block diagram of two degree of freedom QFT control structure.

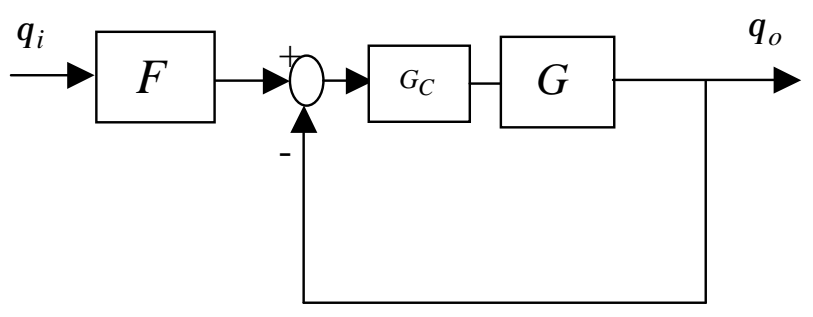

Fig. 6 Two degree of freedom QFT controller structure

The QFT control is used to achieve reliability and robustness, where QFT deals with robust stability margins and robust performance specifications (disturbance rejection, reference tracking, etc). The QFT methodology converts closed-loop system specifications and model plant uncertainty in a set of constraints or bounds for every frequency of interest that will have to be fulfilled by the nominal open loop transfer function. They are represented on a Nichols chart. Such a great integration of information in a set of simple curves will allow designing the controller using only a single plant, which is the nominal plant.

In the design stage (loop-shaping), the controller $G_{C}(s)$ is synthesized by adding poles and zeros until the nominal loop, defined as $L_{O}=G_{O} G_{C}$, lies near its bounds. An optimal controller will be obtained if it meets its bounds and it has the minimum high frequency gain. The general formulation for the controller structure is expressed by the following transfer function:

$$
G_{C}(s)=K_{G} \frac{\prod_{i=1}^{n_{F}}\left(\frac{s}{z_{i}}+1\right) \prod_{i=1}^{n_{c}}{ }^{\prime 2}\left(\frac{s^{2}}{\left|z_{i}\right|^{2}}+\frac{2 \operatorname{Re}\left(z_{i}\right)}{\left|z_{i}\right|^{2}} s+1\right)}{s^{r} \prod_{j=1}^{m_{m}}\left(\frac{s}{p_{j}}+1\right) \prod_{j=1}^{m_{c j}^{\prime \prime 2}}\left(\frac{s^{2}}{\left|p_{j}\right|^{2}}+\frac{2 \operatorname{Re}\left(p_{j}\right)}{\left|p_{j}\right|^{2}} s+1\right)}
$$

Where $K_{G}$ is the gain, $z_{i}$ is a zero that may be complex ( $n_{c z}$, number of complex zeros) or real ( $n_{r z}$, number of real zeros), and $p_{j}$ is a pole (real or complex) with $m_{r p}$ the number of real poles and $m_{c p}$ the number of complex poles $[8,9]$.

\section{CONTROLLER DESIGN}

The closed loop responses can be represented by $T=\frac{\theta_{o}}{\theta_{i}}=\frac{L}{1+L}$ with respect to the desired position of the motor rotor $\left(\theta_{o}\right) . L=G_{C}(s) G(s)$ is the loop transmission and $G_{C}(s)$ is the controller. There are families of $\mathrm{T}$ due to the parametric uncertainty in the transfer function $G(s)$. The objective of the QFT controller is to reduce the variation of the closed loop responses due to the uncertainty in the system. On the other hand it is required to reduce the sensitivity function $S=\frac{1}{1+L}$ to improve the response due to the disturbance. The sensitivity function is chosen to be $|S|<1$ for the range of frequency from 0 to $10 \mathrm{rad} / \mathrm{sec}$. to reject the disturbance at this range of frequency.

The performance specifications to be achieved in this work are robust stability, good tracking and disturbance attenuation. These specifications are used compute the QFT bounds, that guide the shaping of nominal loop. To ensure robust stability of the closed loop system, the following constraint on the peak magnitude of the closed loop frequency response is considered:

$$
\left|\frac{L(s)}{1+L(s)}\right| \leq 1.24
$$

This specification gives minimum gain and phase margins of $5.1 \mathrm{~dB}$ and $47^{\circ}$, respectively.

In order to ensure good disturbance rejection, the sensitivity function should satisfy the performance criterion $|S|<1$ over some frequency range. The range of frequency for disturbance rejection is chosen to be from 0 to $10 \mathrm{rad} / \mathrm{sec}$. and to ensure this performance criterion the sensitivity function is multiplied by a weighting function:

$$
\begin{aligned}
& W_{P}=\frac{0.5 s+10}{s+0.1} \\
& \text { to satisfy }\left|S W_{P}\right|<1
\end{aligned}
$$

The intersection of the QFT bounds was computed on the Nichols chart at a number of design techniques using plant templates derived from Bode plots of the open loop transfer functions. Figure (7) shows the important QFT bounds and the nominal loop transmission. From this figure, the loop transmission was obtained by adjusting the controller gain and choosing the suitable poles and zeros. 


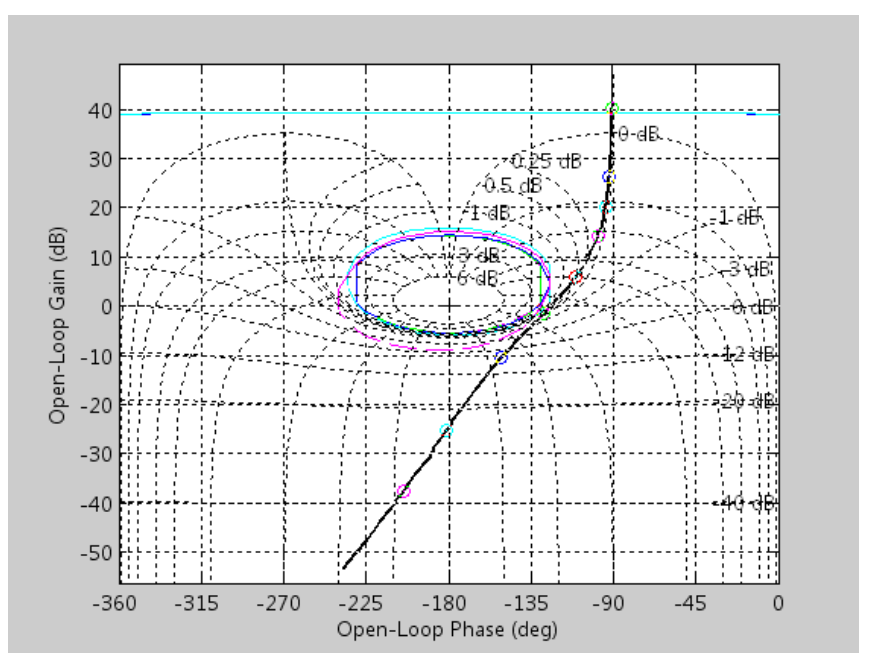

Fig. 7 QFT bounds and nominal loop transmission

The transfer function of the chosen controller is:

$$
G_{C}(s)=\frac{113.3\left(2.12 * 10^{-6} s^{2}+1.6516 * 10^{-4} s+1\right)}{s\left(3.4 * 10^{-6} s^{2}+7.4 * 10^{-3} s+1\right)}
$$

To obtain good reference tracking and while having stability, the prefilter $\mathrm{F}$ was designed using straight line approximation.

$$
F=\frac{1}{0.01245 s+1}
$$

\section{SIMULATION RESULTS}

To verify the design of QFT controller, the overall controlled system is simulated as shown in figures (8a) and (8b) where the closed loop frequency and time response characteristics of the system with the designed controller are obtained.

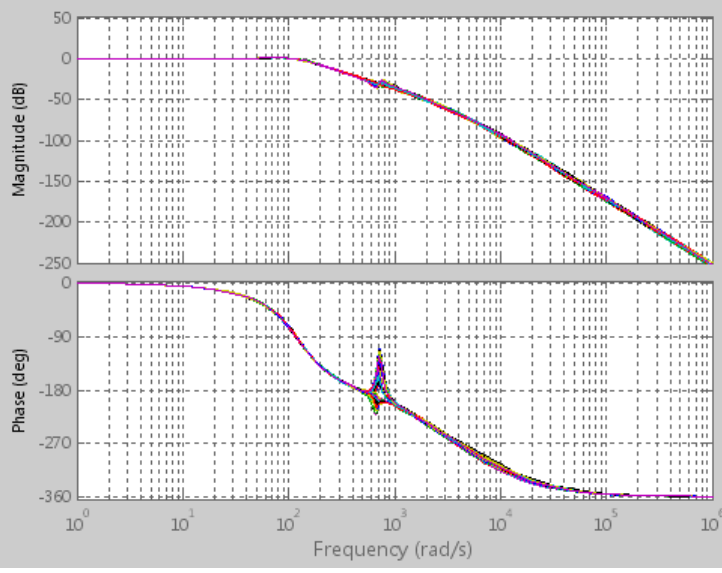

Fig. 8a Closed loop frequency response characteristics of the system with QFT controller

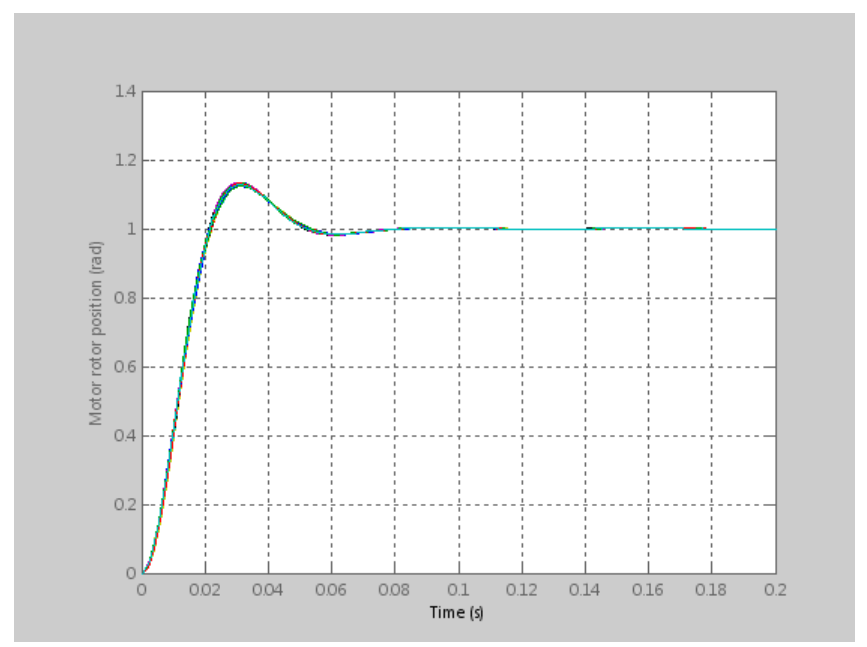

Fig. 8b Closed loop time response characteristics of the system with QFT controller

Figure (9) shows the plot of L, T, and S and it is clear the effect of weighting function on the shape of sensitivity function $\mathrm{S}$.

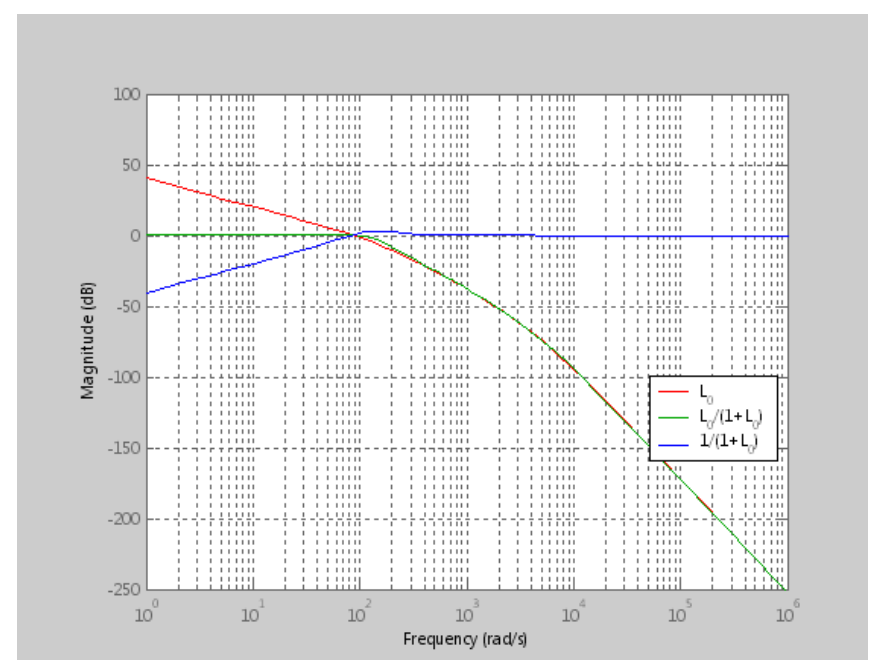

Fig. 9 Plot of the nominal system, complementary sensitivity and sensitivity functions

The results illustrate the robust stability and system disturbance rejection. An improved response with good reference tracking was satisfied after the adding of the prefilter as shown in figures (10) and (11).

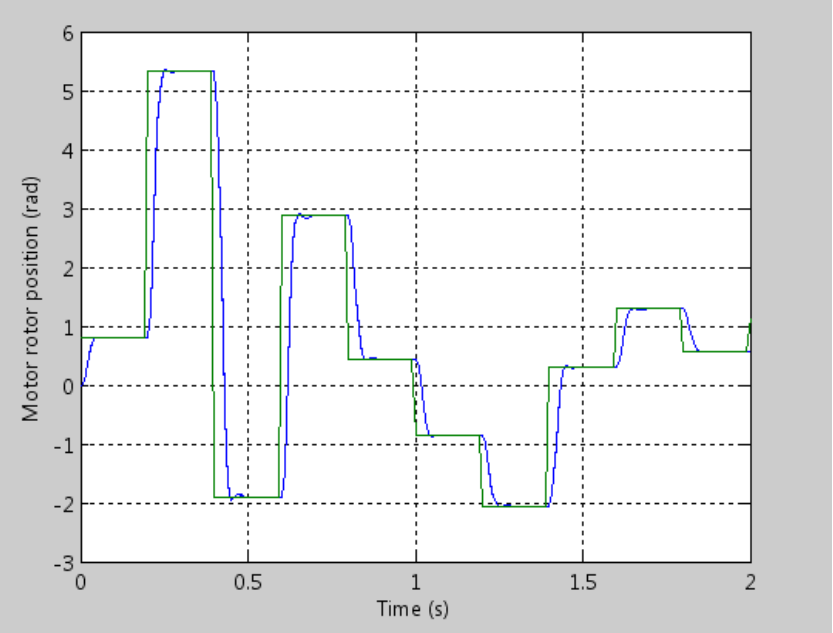


Fig. 10 Closed loop time response for the system with QFT controller and prefilter

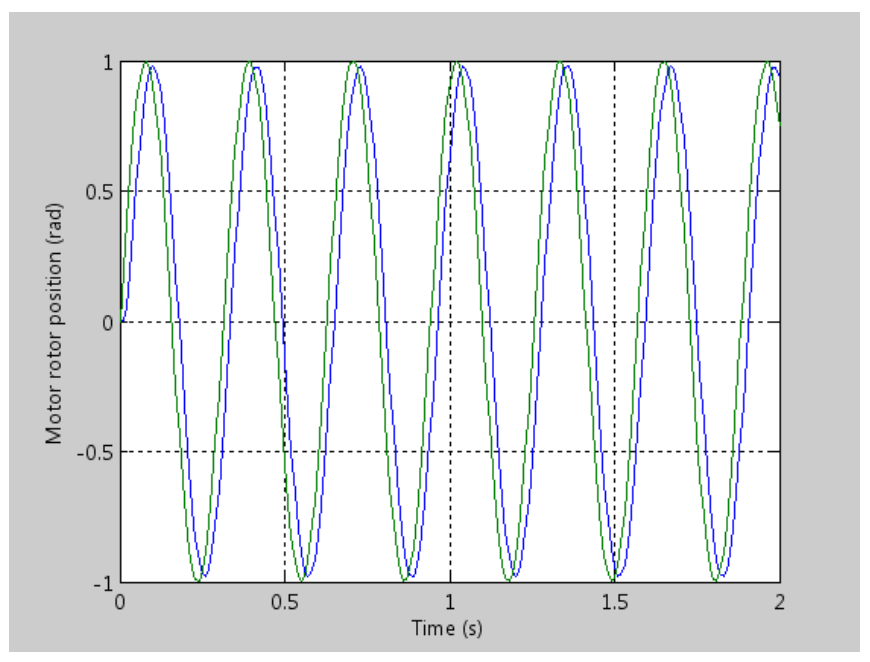

Fig. 11 System performance for sine-input

\section{CONCLUSION}

An application of the QFT controller to a permanent magnet two phase stepping motor has been presented. It was shown that the robust stability and performance (disturbance rejection, reference tracking) were satisfied by applying the QFT controller with the presence of system parameters uncertainty. Also it was clear that the obtained controller was simple, robust and low order. The main objectives of the controller were verified by simulation.

\section{REFERENCES}

[1] Ahmad M. H. and Ashraf. A. Z., Nonlinear control of permanent magner stepper motors, Communications in nonlinear science and numerical simulation, 2004, Vol. 9, pp. 443-458.

[2] Nollet F., Floquet T. and Perruquetti W., Observer-based second order sliding mode control laws for stepper motors, Control engineering practice, 2008, Vol. 16, pp. 429-443.

[3] Acarnley, P., Stepping motors: A guide to modern theory and practice, second edition. Peter Peregrinus Ltd. 1982. 160p.

[4] Kenjo, T. \& Sugawara, A., Stepping Motors and their microprocessor controls, second edition. Clarendon Press. 1994. 279p.

[5] Ljung, L., Glad, T. Modeling of Dynamic Systems. Prentice Hall, Inc. 1994. 361p.

[6] Chrifi-Alaoui, L., Lebrun, A. H., Feedback Control of a Permanent Magnet Stepper Motor. 23rd International Conference on Industrial Electronics, Control and Instrumentation, 1997. Vol. 1. pp. 108-113.

[7] Hydon Switch \& Instrument, Inc. Miniature Motors Product catalogue. High resolution linear actuators. Series 15000 bipolar linear stepper motor.

[8] Horowitz, I., Quantitative feedback theory, IEE Control Theory and Applications, 1982, Vol.. 129, pp. 215-226.

[9] Gera, A., Horowitz, I. M., Optimization of the loop transfer function, International Journal of control, 1980, 31, 2, 389-398, 1980 